\title{
The Solution Structure and Error Estimation for The Generalized Linear Complementarity Problem
}

\author{
Tingfa Yan \\ Middle school of Xiao Bu Ling \\ Tancheng, Shandong, 276134 P.R. China
}

\begin{abstract}
In this paper, we consider the generalized linear complementarity problem (GLCP). Firstly, we develop some equivalent reformulations of the problem under milder conditions, and then characterize the solution of the GLCP. Secondly, we also establish the global error estimation for the GLCP by weakening the assumption. These results obtained in this paper can be taken as an extension for the classical linear complementarity problems.
\end{abstract}

Keywords-GLCP; solution structure; error estimation

\section{INTRODUCTION}

Let mappings $F(x)=M x+p, G(x)=N x+q$. The generalized linear complementarity problem, abbreviated as GLCP, is to find vector $x^{*} \in R^{n}$ such that

$$
F\left(x^{*}\right) \in K, G\left(x^{*}\right) \in K^{0}, F\left(x^{*}\right)^{T} G\left(x^{*}\right)=0,
$$

where $M, N \in R^{m \times n}, p, q \in R^{m}, K$ is a polyhedral cone in $R^{m}$, that is, there exist $A \in R^{s \times m}, B \in R^{t \times m}$, such that

$$
K=\left\{v \in R^{m} \mid A v \geq 0, B v=0\right\} .
$$

It is easy to verify that its polar cone $K^{0}$ assumes the following from

$$
K^{0}=\left\{u \in R^{m} \mid u=A^{T} \lambda_{1}+B^{T} \lambda_{2}, \lambda_{1} \in R_{+}^{s}, \lambda_{2} \in R^{t}\right\} .
$$

The solution set of the GLCP is denoted by $X^{*}$, which is assumed to be nonempty throughout this paper.

The GLCP is a direct generalization of the classical linear complementarity problem (LCP) and a special case of the general variational inequalities problem(GVI)([1]). The GLCP was deeply discussed $[1,2,3,4]$ after the work in [5]. The GLCP plays a significant role in economics, engineering, supply chain network equilibrium, etc. ([6, 7, 8, 9]). For example, the balance of supply and demand is central to all economic systems; mathematically, this fundamental equation in economics is often described by a complementarity relation between two sets of decision variables ([9]). Furthermore, the classical Walrasian law of competitive equilibria of exchange economies can be formulated as a generalized nonlinear complementarity problem in the price and excess demand variables ([7]). At the same time, the GLCP be also found applications in contact mechanics problems, structural mechanics problems, obstacle problems mathematical physics, traffic equilibrium problems, etc ([9]), and has been received much attention of researchers.
Up to now, the issues of solution structure and numerical solution methods for GLCP were fully discussed in the literature (e.g., [2, 3, 4, 5, 10, 11, 12]). To our knowledge, Mangasarian and Shiau ([13]) are the first one who gave the solution structure and error estimation analysis to LCP. Latter, Mathias and Pang ([14]) established the solution structure and global error estimation for the LCP with a P-matrix in terms of the natural residual function, and Mangasarian and Ren gave the same error estimation of the LCP with an $R_{0}$-matrix in [15].

Using the implicit Lagrangian function, Luo et al. ([16]) established a global error estimation for the LCP with a nondegenerate matrix. Obviously, the GLCP is an extension of the LCP, the following questions are posed naturally: How about the error estimation for the GLCP? Can the existing error estimation for the LCP be extended to the GLCP? Thus, this motivates us to extend the solution structure and error estimation conclusions of the LCP to the GLCP.

On the other hand, the error estimation for the GLCP was also fully analyzed (e.g., $[4,10,11])$. This paper is a follow-up to $[4,10]$, as in these papers will establish the global error estimation of the GLCP under weaker conditions than that needed in [4].

The paper is organized as follows. In Section 2, we present some equivalent reformulations of the problem under milder conditions, and detect the solution characterization of the GLCP under milder conditions. The global error estimation is also established for the GLCP in Section 3. Section 4 concludes this paper. These constitute which can be taken as extensions of those for LCP.

Some notations used in this paper are in order. Vectors considered in this paper are all taken in Euclidean space $R^{n}$ equipped with the standard inner product. The 2-norm of vector in the space is denoted by $\|\cdot\|$. We use $R_{+}^{n}$ to denote the nonnegative orthant in $R^{n}$, use $x_{+}$and $x_{-}$to denote the vectors composed by elements

$$
\left(x_{+}\right)_{i}:=\max \left\{x_{i}, 0\right\},\left(x_{-}\right)_{i}:=\max \left\{-x_{i}, 0\right\}, 1 \leq i \leq n,
$$

respectively, and use $\operatorname{dist}\left(x, X^{*}\right)$ to denote the distance from a point $x$ to the solution set $X^{*}$. For simplicity, we also use $x \geq 0$ to denote a nonnegative vector $x \in R^{n}$ if there is no confusion. 


\section{The SOLUTION STRUCTURE FOR GLCP}

In this section, we mainly present the characterization of the solution for GLCP. First, we give the needed assumptions and some known results from [4] for GLCP.

Assumption 1 For $A, M, N$ are the matrices defined in (1).

(A1) The matrix $M^{T} N$ is semi-definite (not necessarily symmetric);

(A2) The matrix $A^{T}$ is column-full rank.

Remark 1. Under Assumption(A2), $A^{T}$ has full-column rank and it has left inverse $\left(A A^{\circ}\right)^{-1} A$, which is also its pesudoinverse of $A^{T}$. On the other hand, the condition that the matrix $A^{T}$ has full-column rank is weaker than that the matrix $\left(A^{*}, B^{*}\right)$ has full-column rank discussed in [4].

Under Assumption (A2), we can establish the following equivalent formulation of the $\operatorname{GLCP}([4])$. i.e., $x$ is a solution of the GLCP if and only if $x$ is a solution of the following system

$$
\left\{\begin{array}{c}
A F(x) \geq 0, \\
B F(x)=0, \\
(F(x))^{\cdot} G(x)=0, \\
U G(x) \geq 0, \\
V G(x)=0,
\end{array}\right.
$$

where

$$
\begin{aligned}
& U=\left\{-A_{L}^{-1} B^{*}\left[\left(A^{\bullet} A_{L}^{-1}-I\right) B^{*}\right]^{+}\left[A^{\bullet} A_{L}^{-1}-I\right]+A_{L}^{-1}\right\}, \\
& V=\left\{A^{*}\left\{-A_{L}^{-1} B^{*}\left[\left(A^{*} A_{L}^{-1}-I\right) B^{*}\right]^{+}\left[A^{*} A_{L}^{-1}-I\right]+A_{L}^{-1}\right\}\right. \\
& \left.+B^{*}\left[\left(A^{*} A_{L}^{-1}-I\right) B^{*}\right]^{+}\left[A^{\cdot} A_{L}^{-1}-I\right]-I\right\} .
\end{aligned}
$$

From (2), using the first equality and the last equality, and combining the first and the second inequality in (2), for any $x \in R^{n}$, we can obtain

$$
\begin{aligned}
& (F(x))^{\cdot} G(x) \\
& =(F(x))^{\bullet}\left\{A ^ { \cdot } \left\{-A_{L}^{-1} B^{\cdot}\left[\left(A^{\cdot} A_{L}^{-1}-I\right) B^{*}\right]^{+}\right.\right. \\
& \left.\times\left[A^{\cdot} A_{L}^{-1}-I\right]+A_{L}^{-1}\right\} \\
& \left.+B^{*}\left[\left(A^{*} A_{L}^{-1}-I\right) B^{*}\right]^{+}\left[A^{*} A_{L}^{-1}-I\right]\right\} G(x) \\
& =[A F(x)]^{*}\left\{-A_{L}^{-1} B^{*}\left[\left(A^{*} A_{L}^{-1}-I\right) B^{*}\right]^{+}\right. \\
& \left.\times\left[A^{\bullet} A_{L}^{-1}-I\right]+A_{L}^{-1}\right\} G(x) \\
& +[B F(x)]^{*}\left\{\left[\left(A^{*} A_{L}^{-1}-I\right) B^{*}\right]^{+}\left[A^{*} A_{L}^{-1}-I\right]\right\} G(x) \\
& =[A F(x)]^{\circ}[U G(x)] \geq 0 .
\end{aligned}
$$

Thus, system (2) can be further written as

$$
\left\{\begin{array}{c}
A F(x) \geq 0, \\
B F(x)=0, \\
(A F(x))^{\cdot}[U G(x)]=0, \\
U G(x) \geq 0, \\
V G(x)=0
\end{array}\right.
$$

Combining (2) with (3), we can establish the following optimization reformulation of the GLCP, and one has that $x^{*}$ is a solution of the GLCP if and only if $x^{*}$ is its global optimal solution with the objective vanishing:

$$
\begin{array}{cc}
\min & H(x)=(M x+p)^{\circ}(N x+q) \\
\text { s.t. } & x \in \Omega,
\end{array}
$$

where $\Omega=\left\{x \in R^{n} \mid A F(x) \geq 0, B F(x)=0, U G(x) \geq 0, V G(x)=0\right\}$.

Under Assumption (A1), $H(x)$ is a convex function, and $\Omega$ is also a convex set. Thus, (5) is a standard convex optimization, we know that its solution set coincides with its stationary point set, i.e., the solution set of the following variational inequality problem: find $x^{*} \in \Omega$ such that

$$
\left(x-x^{*}\right)^{\cdot}\left(\bar{M} x^{*}+\bar{q}\right) \geq 0, \forall x \in \Omega,
$$

where $\bar{M}=M^{*} N+N^{*} M, \bar{q}=M^{*} q+N^{*} p$.

Theorem 1 Suppose that Assumption (A1) and (A2) hold, and $x_{0}$ is a solution of the GLCP. Then

$$
X^{*}=\left\{x \in X \mid \bar{M}\left(x-x_{0}\right)=0,\left(x-x_{0}\right)^{\cdot}\left(\bar{M} x_{0}+\bar{q}\right)=0\right\} .
$$

\section{Proof. Set}

$$
W=\left\{w \in X \mid \bar{M}\left(w-x_{0}\right)=0,\left(w-x_{0}\right)^{\bullet}\left(\bar{M} x_{0}+\bar{q}\right)=0\right\} .
$$

For any $\tilde{x} \in X^{*}$, since $\tilde{x}, x_{0} \in X$, by (6), we get

$$
\begin{aligned}
\left(\tilde{x}-x_{0}\right)^{\cdot}\left(\bar{M} x_{0}+\bar{q}\right) & \geq 0, \\
\left(x_{0}-\tilde{x}\right)^{\cdot}(\bar{M} \tilde{x}+\bar{q}) & \geq 0 .
\end{aligned}
$$

Combining (7) with (8), we can obtain

$$
\left(\tilde{x}-x_{0}\right)^{\bullet} \bar{M}\left(\tilde{x}-x_{0}\right) \leq 0 .
$$

Combining this with Assumption (A1), we get

$$
\left(\tilde{x}-x_{0}\right)^{\bullet} \bar{M}\left(\tilde{x}-x_{0}\right)=0 \text {, }
$$

and conclude that

$$
\bar{M}\left(\tilde{x}-x_{0}\right)=0 .
$$

Combining (9) and (8), one has

$$
\begin{aligned}
\left(x_{0}-\tilde{x}\right)^{\cdot}\left(\bar{M} x_{0}+\bar{q}\right) & =\left(x_{0}-\tilde{x}\right)^{\bullet} \bar{M}\left(x_{0}-\tilde{x}\right) \\
& +\left(x_{0}-\tilde{x}\right)^{\cdot}(\bar{M} \tilde{x}+\bar{q}) \geq 0 .
\end{aligned}
$$

Combining this with (7), we have

$$
\left(\tilde{x}-x_{0}\right)^{\bullet}\left(\bar{M} x_{0}+\bar{q}\right)=0 .
$$

Combining this with (10), we obtain $\tilde{x} \in W$.

On the other hand, for any $w \in W$, since

$$
\bar{M}\left(w-x_{0}\right)=0,\left(w-x_{0}\right)^{\cdot}\left(\bar{M} x_{0}+\bar{q}\right)=0,
$$

for any $x \in X$, using the fact that $x_{0}$ is a solution of the GLCP , combining (6), we obtain

$$
\begin{aligned}
& (x-w)^{\bullet}(\bar{M} w+\bar{q}) \\
& =\left[\left(x-x_{0}\right)-\left(w-x_{0}\right)\right]^{\cdot}\left[\bar{M}\left(w-x_{0}\right)+\left(\bar{M} x_{0}+\bar{q}\right)\right] \\
& =\left[\left(x-x_{0}\right)-\left(w-x_{0}\right)\right]^{\cdot}\left[\left(\bar{M} x_{0}+\bar{q}\right)\right] \\
& =\left(x-x_{0}\right)^{\cdot}\left(\bar{M} x_{0}+\bar{q}\right)-\left(w-x_{0}\right)^{\cdot}\left(\bar{M} x_{0}+\bar{q}\right) \\
& =\left(x-x_{0}\right)^{\cdot}\left(\bar{M} x_{0}+\bar{q}\right) \geq 0,
\end{aligned}
$$

Thus, $w \in X^{*}$.

Theorem 2 If $x_{1}$ and $x_{2}$ are two solutions of the GLCP. Then, $\left(M x_{1}+p\right)^{\circ}\left(N x_{2}+q\right)=\left(M x_{2}+p\right)^{\circ}\left(N x_{1}+q\right)=0$. 
Proof. Since $x_{1}$ and $x_{2}$ are two solutions of the GLCP. By Theorem 1, we have

$$
\bar{M}\left(x_{1}-x_{2}\right)=\bar{M}\left(x_{1}-x_{0}\right)-\bar{M}\left(x_{2}-x_{0}\right)=0 .
$$

Combining this with the fact that $x_{1}$ and $x_{2}$ are two solutions of the GLCP, we have

one has

$$
\left(M x_{1}+p\right)^{\cdot}\left(N x_{1}+q\right)=0,\left(M x_{2}+p\right)^{\cdot}\left(N x_{2}+q\right)=0,
$$

$$
\begin{aligned}
0 & =\left(x_{1}-x_{2}\right)^{\bullet}\left(M^{\bullet} N+N^{*} M\right)\left(x_{1}-x_{2}\right) \\
& =2\left(x_{1}-x_{2}\right)^{\bullet} M^{\cdot} N\left(x_{1}-x_{2}\right) \\
& =2\left[\left(M x_{1}+p\right)-\left(M x_{2}+p\right)\right]^{\bullet}\left[\left(N x_{1}+q\right)-\left(N x_{2}+q\right)\right] \\
& =-2\left[\left(M x_{1}+p\right)^{\cdot}\left(N x_{2}+q\right)+\left(M x_{2}+p\right)^{\cdot}\left(N x_{1}+q\right)\right] .
\end{aligned}
$$

Using the similar technique to that of (3), we can deduce

$$
\left(M x_{1}+p\right)^{\bullet}\left(N x_{2}+q\right) \geq 0,\left(M x_{2}+p\right)^{\bullet}\left(N x_{1}+q\right) \geq 0 .
$$

Combining (11) with (12), and the desired result follows.

Theorem 3 The solution set of GLCP is a convex set.

Proof. If solution set of the GLCP is single point set, then it is obviously convex. In this following, we suppose that $x_{1}$ and $x_{2}$ are two solutions of the GLCP. By Theorem 1, we have

$$
\begin{aligned}
& \bar{M}\left(x_{1}-x_{0}\right)=0, \bar{M}\left(x_{2}-x_{0}\right)=0, \\
& \left(\bar{M} x_{0}+\bar{q}\right)^{T}\left(x_{1}-x_{0}\right)=0 \\
& \left(\bar{M} x_{0}+\bar{q}\right)^{T}\left(x_{2}-x_{0}\right)=0
\end{aligned}
$$

For vector $x=\tau x_{1}+(1-\tau) x_{2}, \forall \tau \in[0,1]$, by (13), we have

$$
\begin{aligned}
& \begin{aligned}
\bar{M}\left(x-x_{0}\right) & =\bar{M}\left[\tau x_{1}+(1-\tau) x_{2}-x_{0}\right] \\
& =\tau \bar{M}\left(x_{1}-x_{0}\right)+(1-\tau) \bar{M}\left(x_{2}-x_{0}\right) \\
& =0 .
\end{aligned} \\
& \left(\bar{M} x_{0}+\bar{q}\right)^{T}\left(x-x_{0}\right) \\
& =\left(\bar{M} x_{0}+\bar{q}\right)^{T}\left[\tau x_{1}+(1-\tau) x_{2}-x_{0}\right] \\
& =\tau\left(\bar{M} x_{0}+\bar{q}\right)^{T}\left(x_{1}-x_{0}\right) \\
& \quad+(1-\tau)\left(\bar{M} x_{0}+\bar{q}\right)^{T}\left(x_{2}-x_{0}\right)=0 .
\end{aligned}
$$

Combining (14), (15) with the conclusion of Theorem 1, we obtain the desired result.

\section{THE ERROR ESTIMATION FOR GLCP}

In this section, we will present a global error estimation for the GLCP under weaker conditions than that needed in [4]. Firstly, we can give the needed error bound for a polyhedral cone from [17] and error bound for a convex optimization from [18] to reach our aims.

Lemma 1 For polyhedral cone

$$
P=\left\{x \in R^{n} \mid D_{1} x=d_{1}, B_{1} x \leq b_{1}\right\}
$$

with $D_{1} \in R^{l \times n}, \quad B_{1} \in R^{m \times n}, \quad d_{1} \in R^{l}$ and $b_{1} \in R^{m}$, there exists a constant $c_{1}>0$ such that $\operatorname{dist}(x, P) \leq c_{1}\left[\left\|D_{1} x-d_{1}\right\|+\left\|\left(B_{1} x-b_{1}\right)_{+}\right\|\right], \quad \forall x \in R^{n}$.

Lemma 2 Let $P$ be a convex polyhedron in $R^{n}$ and $\theta$ be a convex quadratic function defined on $R^{n}$. Let $S$ be the nonempty set of globally optimal solutions of the program:

$$
\min \theta(x)
$$$$
\text { s.t. } \quad x \in P
$$

with $\theta_{o p t}$ being the optimal value of $\theta$ on $S$. There exists a scalar $c_{2}>0$ such that

$$
\begin{aligned}
& \operatorname{dist}(x, S) \leq c_{2} \max \left\{\operatorname{dist}(x, P),\left|\left[\theta(x)-\theta_{o p t}\right]_{+}\right|,\right. \\
&\left.\left|\left[\theta(x)-\theta_{o p t}\right]_{+}\right|^{(1 / 2)}\right\}, \forall x \in R^{n} .
\end{aligned}
$$

Theorem 4 Under Assumption 2.1 (A1) and (A2), then there exists constant $\rho>0$ such that

$$
\begin{aligned}
\operatorname{dist}\left(x, X^{*}\right) \leq \rho\{ & \left\|[A F(x)]_{-}\right\|+\|B F(x)\|+\left\|[U G(x)]_{-}\right\| \\
+ & \|V G(x)\|+\left|\left[F(x)^{\cdot} G(x)\right]_{+}\right| \\
& \left.+\left|\left[F(x)^{\cdot} G(x)\right]_{+}\right|^{(1 / 2)}\right\}, \forall x \in R^{n} .
\end{aligned}
$$

Proof. For problem (5), under Assumption 2.1 (A1), $H(x)$ is a convex function, and we know that $x^{*}$ is a solution of the GLCP if and only if $x^{*}$ is its global optimal solution with the objective vanishing, i.e., $H(x)_{o p t}=0$. For any $x \in R^{n}$, a direct computation yields that

$$
\begin{aligned}
\operatorname{dist}\left(x, X^{*}\right) \leq & c_{3} \max \left\{\operatorname{dist}(x, \Omega),\left|[H(x)]_{+}\right|,\left|[H(x)]_{+}\right|^{(1 / 2)}\right\} \\
\leq & c_{3} \max \left\{c_{4} \mid\left\|[A F(x)]_{-}\right\|+\|B F(x)\|+\left\|[G(x)]_{-}\right\|\right. \\
& \left.+\|V G(x)\|\},\left|[H(x)]_{+}\right|,\left|[H(x)]_{+}\right|^{(1 / 2)}\right\} \\
\leq & c_{3} \max \left\{c_{4}, 1\right\}\left\{\left\|[A F(x)]_{-}\right\|+\|B F(x)\|\right. \\
& +\left\|[U G(x)]_{-}\right\|+\|V G(x)\| \\
& \left.+\left|\left[(F(x))^{\cdot} G(x)\right]_{+}\right|+\left|\left[(F(x))^{\cdot} G(x)\right]_{+}\right|^{(1 / 2)}\right\},
\end{aligned}
$$

where the first inequality follows from Lemma 2 with constant $c_{3}>0$, and the second inequality uses Lemma 1 with constant $c_{4}>0$. Using (16), letting $\rho=c_{3} \max \left\{c_{4}, 1\right\}$, the desired result follows.

Remark 2. Combining Remark 1. Assumption 1 (A2) in Theorem 4 is weaker than the Assumption (A2) in Theorem 4.1 in [4], and the Assumption (A1) in this paper coincides with Assumption (A1) in [4]. In addition, Theorem 4 is sharper than Theorem 4.1 in [4].

In the end of this paper, we will consider a special case of GLCP which was discussed in $[13,14,15,16]$.

When $K=R_{+}^{n}, F(x)=x$, then $K^{0}=R_{+}^{n}$, and GLCP reduces to the LCP of finding vector $x^{*} \in R^{n}$ such that

$$
x^{*} \geq 0, N x^{*}+q \geq 0,\left(x^{*}\right)^{T}\left(N x^{*}+q\right)=0 .
$$


Combining (17) with Theorem 4, we can immediately obtain the following conclusion.

Corollary 1 Suppose the matrix $N$ is semi-definite (not necessarily symmetric), and $F(x)=x$. Then there exists constant $\rho_{1}>0$ such that

$$
\begin{gathered}
\operatorname{dist}\left(x, X^{*}\right) \leq \rho_{1}\left\{\left\|x_{-}\right\|+\left\|(N x+q)_{-}\right\|+\left|\left[x^{*}(N x+q)\right]_{+}\right|\right. \\
\left.+\left|[x(N x+q)]_{+}\right|^{(1 / 2)}\right\}, \forall x \in R^{n} .
\end{gathered}
$$

Proof. By $K=R_{+}^{n}$, we have $A=I, B=0$, from $F(x)=x$, we have $M=I, q=0$, where $I$ is an identity matrix. Combining this with definition of $U, V$ in (2), we can obtain $U=I, V=0$. Combining these with Theorem 4, then the desired result follows.

Remark 3. It is clear that the assumption in Corollary 1 above coincides with that in Theorem 2.7 in [13]. Furthermore, the conclusion in Corollary 1 is stronger than that in Theorem 2.7 in [13].

When $K=R_{+}^{n}$, GLCP reduces to the vertical linear complementarity problem([19])of finding vector $x^{*} \in R^{n}$ such that

$$
M x^{*}+q \geq 0, N x^{*}+q \geq 0,\left(M x^{*}+q\right)^{T}\left(N x^{*}+q\right)=0 .
$$

Combining (18) with Theorem 4, we have the following conclusion hold.

Corollary 2 Under Assumption 2.1 (A1). Then there exists constant $\rho_{2}>0$ such that

$$
\begin{aligned}
\operatorname{dist}\left(x, X^{*}\right) \leq & \rho_{2}\left\{\left\|(M x+p)_{-}\right\|+\left\|(N x+q)_{-}\right\|\right. \\
& +\left|\left[(M x+p)^{\cdot}(N x+q)\right]_{+}\right| \\
& \left.+\left|\left[(M x+p)^{\cdot}(N x+q)\right]_{+}\right|^{(1 / 2)}\right\}, \forall x \in R^{n} .
\end{aligned}
$$

Proof. By $K=R_{+}^{n}$, one has $A=I, B=0$. Combining this with definition of $U, V$ in (2), one has $U=I, V=0$. Combining these with Theorem 4, then the desired result follows.

Remark 4. Obviously, Assumption 2.1(A1) in Corollary 2 above is weaker than that in Corollary 2 in [19], since the condition which rank $\left(M^{T}, N^{T}\right)^{T}=n$ is removed.

\section{CONCLUSIONS}

In this paper, we presented the solution characterization for GLCP, and established global error estimation on the GLCP under weaker conditions than that needed in [4], which is the extension of this for LCP. Surely, under milder conditions, we may also established the solution structure and error estimation for GLCP such as the mapping being nonmonotone involved in the GLCP, this is a topic for future research.

\section{ACKNOWLEDGMENT}

This work was supported by the Logistics Teaching and Research Reformation Projects for Chinese Universities (JZW2014048, JZW2014049), the Shandong Province Science and Technology Development Projects (2013GGA13034), the Natural Science Foundation of Shandong Province (ZR2011FL017).

\section{REFERENCES}

[1] M.A. Noor, "General variational inequalities", Appl. Math. Letters, 1(2), pp. 119-121, 1988.

[2] Y.J. Wang, F.M. Ma, J.Z. Zhang, "A nonsmooth L-M method for solving the generalized nonlinear complementarity problem over a polyhedral cone", Appl. Math. Optim., 52, pp. 73-92, 2005.

[3] X.Z. Zhang, F.M. Ma, Y.J. Wang, "A Newton-type algorithm for generalized linear complementarity problem over a polyhedral cone", Appl. Math. Comput., 169, pp. 388-401, 2005

[4] H. C. Sun, Y.J. Wang, "Further discussion on the error bound for generalized linear complementarity problem over a polyhedral cone", J. Optim. Theory Appl., 159(1),pp.93-107, 2013.

[5] R. Andreani, A. Friedlander, S.A. Santos, "On the resolution of the generalized nonlinear complementarity problem”, SIAM J. Optim., 12, pp. 303-321, 2001.

[6] F. Facchinei and J.S. Pang, Finite-dimensional variational inequality and complementarity problems, Springer, New York, NY, 2003.

[7] L. Walras , Elements of pure economics, Allen and Unwin, London, 1954

[8] L.P. Zhang, "A nonlinear complementarity model for supply chain network equilibrium", Journal of Industrial and Managment Optimization, 3(4), pp.727-737, 2007.

[9] M.C. Ferris, J.S. Pang, "Engineering and economic applications of complementarity problems", Society for industrial and applied mathematics, 39(4), pp.669-713, 1997.

[10] Y. Z. Diao, "An error estimation for management equilibrium model", International Journal of Computer and Information Technology, 2(4), pp.677-681,2013.

[11] S.S. Xie, P. Wang, L. Wang, H.C. Sun, “An algorithm for the nonlinear complementarity problem on management equilibrium model", International Journal of Computer and Information Technology, 2(6), pp.1136-1140, 2013.

[12] L. Wang, "A global convergence algorithm for the supply chain network equilibrium model", International Journal of Advanced Computer Science and Applications, 3(2), pp.15-18,2012.

[13] O.L. Mangasarian and T.H. Shiau, "Error bounds for monotone linear complementarity problems", Math. Programming, 36(1): 81--89, 1986.

[14] R. Mathias and J.S. Pang, "Error bound for the linear complementarity problem with a P-matrix", Linear Algebra \& Appl., 132: 123-136, 1990.

[15] O.L. Mangasarian and J. Ren, "New improved error bound for the linear complementtarity problem", Math. Programming, 66: 241-255, 1994.

[16] Z.Q. Luo, O.L. Mangasarian, J. Ren and M.V. Solodov, “ New error bound for the linear complementarity problem”, Math. Operations Research, 19: 880-892, 1994.

[17] A.J. Hoffman, "On the approximate solutions of linear inequalities", Journal of Research of the National Bureau of Standards, 49, pp. 263$265,1952$.

[18] T. Wang and J.S. Pang, "Global error bounds for convex quadratic inequality systems", Optimization, 31, pp. 1-12, 1994.

[19] J.Z. Zhang, N.H. Xiu, "Global s-type error bound for the extended linear complementarity problem and applications" , Math. Program., Ser. B, 88: 391-410, 2000 . 